\title{
RESONANCE IONIZATION SPECTROSCOPY AND ITS APPLICATION
}

\author{
H.-J. KLugE \\ Institut für Physik, Universität Mainz, 55099 Mainz, Germany
}

\begin{abstract}
Resonance ionization spectroscopy and its combination with mass spectrometry offer very high sensitivity and selectivity. The application of several variants of resonance ionization mass spectroscopy to the study of nuclear ground statc properties and trace analysis of radioactive isotopes in the environment is discussed as well as the inverse process, i.e. the laser induced recombination, of bare heavy ions stored at relativistic energies in storage rings.
\end{abstract}

PACS numbers: $32.80 . \mathrm{Fb}$

\section{Introduction}

Resonance ionization spectroscopy (RIS) was proposed as early as 1972. By use of resonant laser light at different wave lengths the atom is stepwise sxcited and finally ionized. Such a scheme has a number of advantages:

1. An ion and/or electron is detected. This can be done with much higher efficiency than in the case of detecting photons. Ilence, RIS is a very sensitive technique, allowing for the detection and investigation of very rare or exotic atoms.

2. The resonance condition in each excitation step provides high elemental and isotopic selectivity. The problem of interference by contaminations can be avoided, which is of major concern in conventional optical trace analysis. Furthermorc, isobaric interference as observed in mass spectrometry is strongly reduced.

3. The selectivity of RIS can still be enhanced by a mass-selective detection of the ion created in resonance. The combination of RIS with mass spectrometry is called RIMS (resonance ionization mass spectroscopy).

4. The background can be kept low, due to the use of detectors for charged particles. 
In the last 20 years the RIS technique has been more and more refined, profiting also from the development of ever more powerful lasers. It has been applied to a large number of exotic particles, isotopes, elements, and molecules in many arcas of science. The achieved performance is documented in books $[1,2]$ and the proccedings of the conference series "Resonance Ionization Spectroscopy and its Applications" [3].

Four different RIS technique experiments will be presented in the following after a short discussion of the principle of resonance ionization. These examples concern problems in nuclear physics, atomic physics, cosmology, and chemistry. In the last section, the inverse resonance ionization process, i.e. the laser-induced recombination, will be briefly discussed, which has just recently been observed at storage rings.

\section{Principle of resonance ionization spectroscopy}

Figure 1 shows the scheme for resonance ionization: Thrce-colour, two- or three-step resonant excitation is taken as an example. The three schemes differ in the last step, the ionization step. This represents usually the bottle-neck of the excitation path due to the low cross section for non-resonant photo-ionization (left) which is of the order of $\sigma=10^{-17} \div 10^{-19} \mathrm{~cm}^{2}$. Two to four orders in cross section can be gained by exciting to a Rydberg level (middle) which is ionized by an electric field, collisions with gas molecules or a fourth (infrared) laser beam, or to an auto-ionizing state (right). Since the electron has to be promoted to the next excited atomic level before decay by resonance fluorescence takes place $\left(\tau \approx 10^{-8} \mathrm{~s}\right)$, pulsed tunable lasers have to be applied. But in selected cases, where optical pumping can be avoided and small laser bandwith is required, also cw-tunable lasers might be applied.

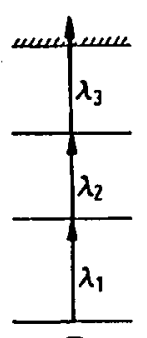

(1)

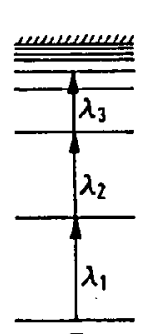

(b)

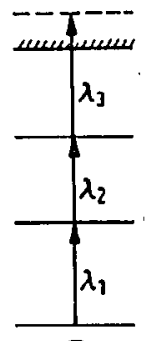

(c)

Fig. 1. Scheme of resonance ionization spectroscopy for the case of a three-colour excitation to the continuum (left), to a high-lying Rydberg level (middle), or to an auto-ionizing state (right).

The elemental selectivity of resonance ionization spectroscopy of atoms is cxtremely high because of the low level density in atoms which can be reached by allowed elcctric dipole transitions. Typically, the chance to hit a resonance of another atom is of the order of $10^{-5}$ to $10^{-6}$. If more than onc resonant, stepwise 
excitation is induced as in Fig. 1, the selectivities in the different resonance transitions can be multiplied to obtain the total selectivity in the RIS process. In the case of RIMS, where a mass spectrometer is added with an enhancement factor ranging from $10^{3}$ to $10^{6}$, the total elemental selectivity is comparable or surpasses the selectivity of tandem mass spectrometry, the most selective technique known today. IIence, isobaric contaminations, as usually observed in mass spectrometry, are extremely sufficiently suppressed by RIMS.

In the case of isotopic selectivity, the situation is usually different. IIere, the hyperfine structure (IIFS) and isotope shift (IS) are generally too small in order to achieve efficient discrimination against isotopes of the same element. This is especially true if pulsed laser systems with broader laser bandwiths are applied. In such cases, a pulsed laser system offers the cheap but efficient possibility to add a time-of-flight (TOF) mass spectrometer. TOF systems enable an abundance sensitivity of about $10^{3}$ to $10^{4}$. Again, an increase by at least two orders of magnitude can be achieved by use of magnetic mass spectrometers. If still higher isotopic selectivity is required, one may induce an artificial isotopic mass shift as in the strontium experiment described below. If the separation $\Delta \nu$ between the resonances of the isotope under investigation and the interfering one is much larger than the linewidth $\Gamma=1 /(2 \pi \tau)$, then the selectivity is given by

$$
S=(2 \times \Delta \nu / \Gamma)^{2} \text {. }
$$

The excitation path for RIS has to be chosen careful in respect to a vailable laser systems, the possibility to saturate the optical transition (sensitivity) and possible interferences (background). The task generally involves the determination of cross sections, lifetimes, hyperfine structure splittings, isotope shifts, and the search for auto-ionizing states.

\section{Resonance ionization spectroscopy. Experiments and results}

Four experiments will be described in the following. Since experimental details and most of the results are published, the chapter tries to give an $0^{\prime \prime}$ erview. The reader is referred to the original publications.

\subsection{Laser spectroscopy of neutron-deficient gold and platinum isotopes}

In 1972, the first systematic investigation of a long isotopic series by atomic spectroscopy led to a completely unexpected result: Between ${ }^{187} \mathrm{Hg}$ and ${ }^{185} \mathrm{IIg}$ a sudden break in the trend of the isotope shifts and, hence, in the mean square charge radii was observed by Bonn et al. [4]. This break corresponds to the addition of 11 neutrons. It was explained as due to a sharp nuclear shape transition: The nuclei with $A \geq 187$ are slightly oblate, whereas the odd nuclei with $A \leq 187$ are strongly prolate deformed with a deformation parameter of $\beta \geq 0.25$. This discovery offered the key for interpreting the structure of the nuclei in this region as caused by nuclear shape coexistence. This effect is also manifested in the observation of the coexistence of two different shapes at almost degenerated energies in ${ }^{185} \mathrm{Hg}$ and in the largest odd-even staggering of nuclear charge radii ever observed (Fig. 2). 


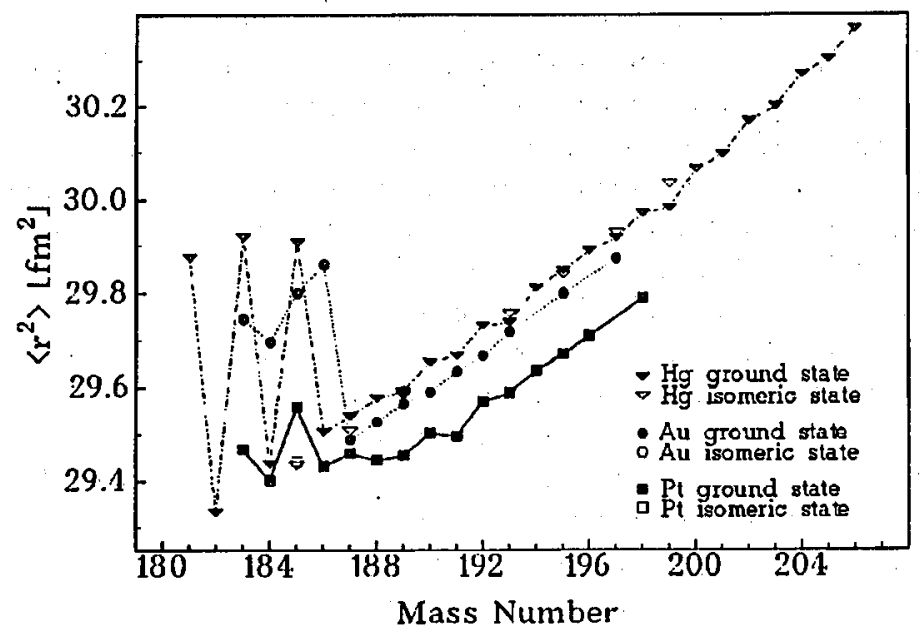

Fig. 2. Changes of mean-square charge radii in the isotopic sequences of $\mathrm{Hg}, \mathrm{Au}$, and $\mathrm{Pt}$ as a function of mass number. The radii of ground states are indicated and connected by eye-guiding lines. In addition, the charge radii of isomeric states are given. The errors are smaller than the diameter of the symbols except for ${ }^{183} \mathrm{Pt}$ and ${ }^{184} \mathrm{Au}$.

This observation stimulated the investigation of isotopes in the very same mass region. ( $A \approx 185$, mid-shell neutron number) of the neighbouring elements gold [5] and platinum [6]. Both are refractory elements which are not or only very recently $(\mathrm{Au})$ available at the ISOLDE facility/CERN as on-line ion beams. $\Lambda$ new technique was developed which is RIMS in combination with pulsed laser induced desorption (RIMS/PLID) [7].

Figure 3 shows the experimental set-up installed [7]. A mass-separated IIg ion beam is implanted into a graphite target. After decay to the $\mathrm{Au}$ or $\mathrm{Pl}$ daughter, the target wheel is turned by $180^{\circ}$ and the radioactivity is released as pulsed thermal beam by laser desorption. The atomic beam is then stepwise resonantly excited and finally ionized by the light of three pulsed tunable dye lasers, synchronized with the pulse of the desorption laser. Hence, good temporal overlap is obtained between the pulsed atomic beam and the pulsed laser beams for RIS. The photo ions created in resonance are detected mass-selectively by a TOF spectrometer. This completes the method to resonance ionization mass spectrometry.

Figure 2 shows the charge radii of the Au and $\mathrm{Pt}$ isotopes measured by RIMS together with the corresponding data on mercury. These results show the usual shrinking of the nucleus as a function of decreasing neutron number. In the case of $\mathrm{Au}$, only one drastic change in deformation is observed due to the stabilizing power of the unpaired proton. In the isolopic chain of $\mathrm{Pt}$ no indication of a sharp break is obtained. Nevertheless, an analysis of the IS of Pt shows that a similar deformation $(\beta=0.24)$ is observed for ${ }^{183} \mathrm{Pt}$ as in the isobaric and isctonic $\mathrm{Au}$ and Pt nuclei. 


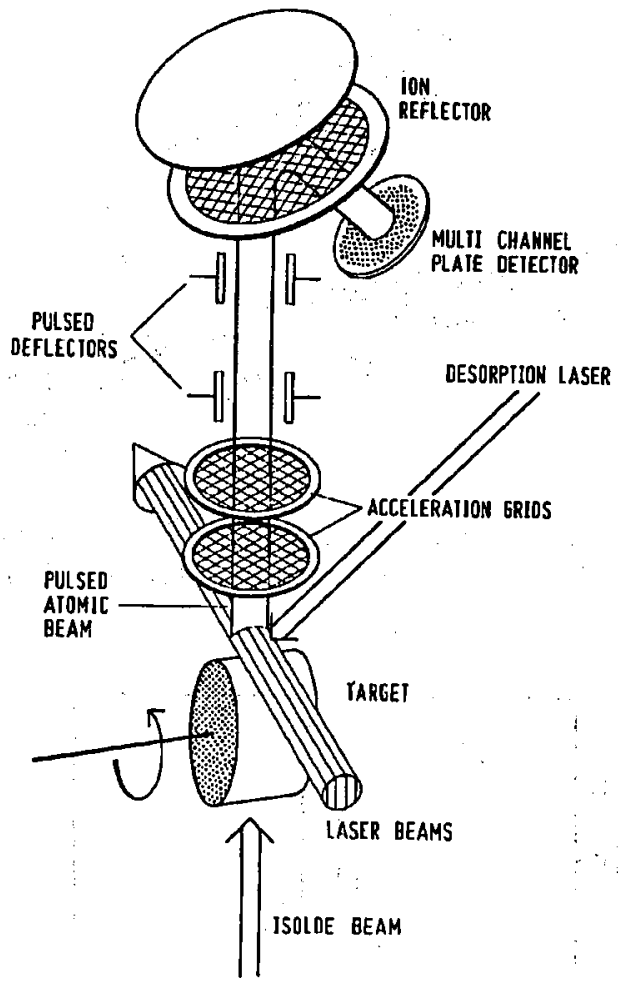

Fig. 3. Schematic diagram of resonance ionization mass spectroscopy in combination with pulsed laser-induced desorption for the investigation of $\mathrm{Au}$ and $\mathrm{Pt}$ isotopes.

\subsection{Resonance ionization spectroscopy on a fast alomic beam in collinear geometry; trace detection of ${ }^{89,90} \mathrm{Sr}$}

A challenge for trace analysis is the fast and sensitive detection of the radioactive isotope ${ }^{89} \mathrm{Sr}$ and ${ }^{90} \mathrm{Sr}$ spread out in nuclear weapon tests or in case of reactor accidents. Especially the long-lived isotope ${ }^{90} \mathrm{Sr}\left(T_{1 / 2}=28.5 \mathrm{a}\right)$ is dangerous as it is accumulated in the human bones as chemical equivalent of calcium. The radiochemical detection of ${ }^{90} \mathrm{Sr}$ is slow (in the order of two weeks), because it is detected via the $\beta$-decay of the daughter nucleus, ${ }^{90} \mathrm{Y}$. In order to get immediate information on the pollution by ${ }^{90} \mathrm{Sr}$ in an accident such as Chernobyl, a non-radiative detection method is required. Since the pollution is firstly transported by air, air samples should be investigated. A typical sample as extracted from $1000 \mathrm{~m}^{3}$ air immediately after the Chernobyl accident in Germany contained only about $10^{8}$ atoms of ${ }^{89,90} \mathrm{Sr}$ but up to $10^{18}$ atoms of stable strontium. Therefore, an extremely high isotopic selectivity of $S>10^{10}$ is required. At the same time, the detection efficiency must be high for reasonable statistics and the total measuring time including the sample preparation should not exceed one day.

These demands can be met by RIS on a fast atomic beam in collinear geometry by use of cw laser light for excitation [8]. Figure 4 shows the set-up. The $\mathrm{Sr}$ 


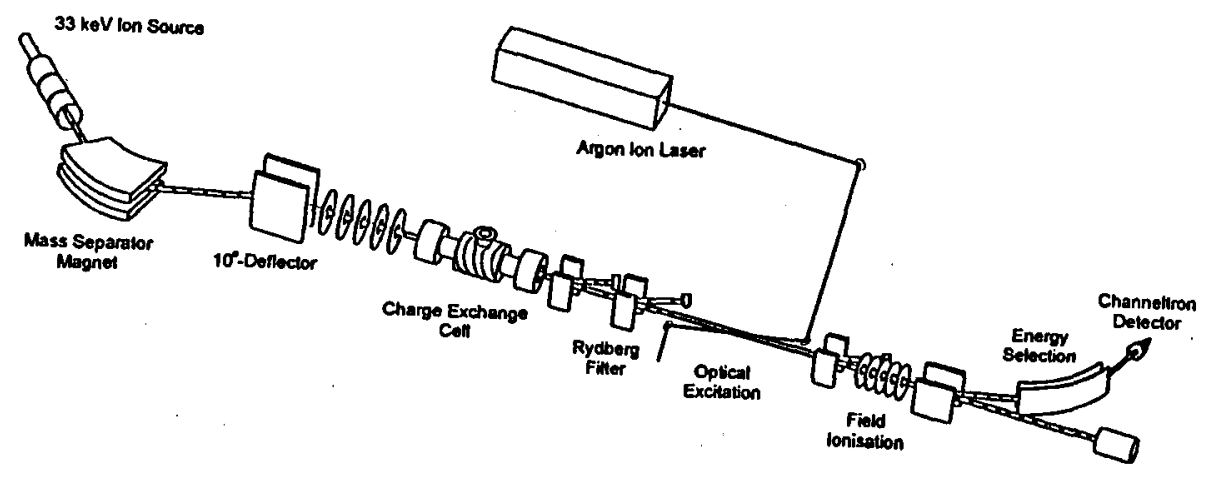

Fig. 4. Experimental set-up for trace analysis of ${ }^{90} \mathrm{Sr}$ by resonance ionization in. collinear spectroscopy. The angle betwcen laser and fast atomic beam is strongly exaggerated.

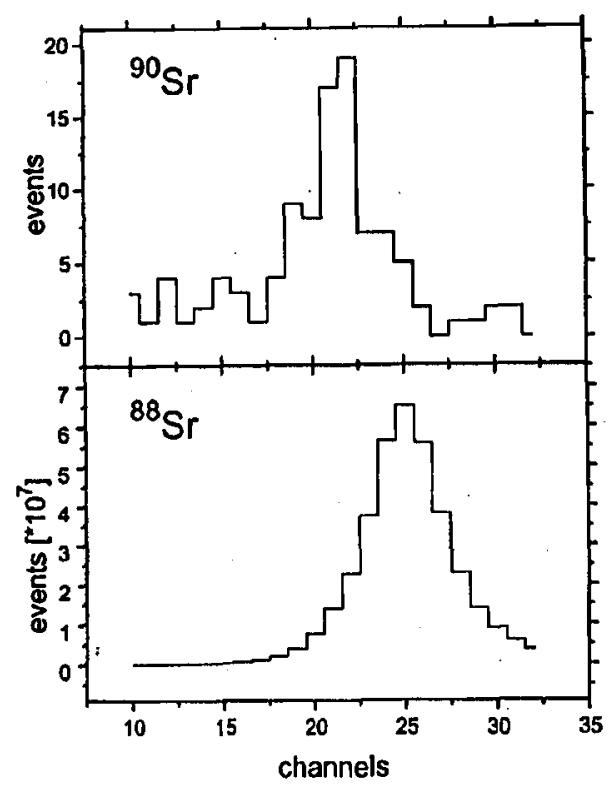

Fig. 5. ${ }^{90} \mathrm{Sr}$ signal (top) from an air sample of about $900 \mathrm{~m}^{3}$ taken immediately after the Chernobyl accident near Munich and the reference signal of ${ }^{88} \mathrm{Sr}$ (bottom).

sample is chemically extracted out of an air sample and put into a surface ionization source. The ions are accelerated to about $32 \mathrm{kV}$, mass separated, and charge exchanged in a cesium vapour cell.

In the charge exchange process the lowest ${ }^{3} P$ and ${ }^{3} D$ states of the strontium atoms are populated. Starting from the ${ }^{3} D_{2}$ state, the fast atoms are excited to $5 \operatorname{snf}{ }^{3} F$-Rydberg states by use of the $\lambda=362 \mathrm{~nm}$ line of an argon ion laser. Tunability is achieved by changing the acceleration voltage of the ion beam. The 
laser excited Rydberg atoms are field ionized, deflected from the neutral beam, energy analyzed in an clectrostatic deflector and finally counted by a channeltron detector.

The background in this experiment is mainly due to Rydberg atoms produced in the charge exchange process and by collisions with the residual gas. Therefore, three ion filters are installed in the atomic beam path. At the first Rydberg filter a rather high voltage is applied in order to ionize and deflect Rydberg atoms produced in the charge exchange process. In order to avoid a reduction of the optical selectivity by the Stark effect in the electric field of these filters, the laser beam is not completely collinearly superimposed to the atomic beam but in a small angle. In this way the excitation of the strontium atoms takes place in a field-free region only, while the advantage of the large artificial mass shift is fully conserved. Figure 5 shows the example of a measurement of an environmental sample taken shortly after the Chernobyl accident. An amount of $3.6 \times 10^{8}$ atoms of ${ }^{90} \mathrm{Sr}$ could be detected in the presence of $10^{17}$ atoms of stable strontium. Presently, the detection limit of this technique is about $5 \times 10^{7}$ atoms of ${ }^{90} \mathrm{Sr}$.

\subsection{Spectroscopy and trace analysis of technetium, plutonium and olher actinides}

Up to now, spectroscopy and trace analysis of these elements released in accidents of nuclear power plants or in atomic bomb tests are rather limited due to the high toxicity and radioactivity. Radiochemical techniques have usually a detection limit between $10^{8}$ to $10^{10}$ atoms at a very long measuring time. Furthermore, it is not possible to determine the isotopic composition of the sample with these techniques.

The experimental set-up is shown in Fig. 6. Three tunable dye lasers are pumped by two pulsed copper vapour lasers at a repetition rate of $6.5 \mathrm{kHz}$. The dye laser beams are injected into a time-of-flight spectrometer of linear type [9] or of reflectron type (Fig. 6) with the help of prisms or by coupling the light into optical fibres. The ions produced by resonance ionization in the interaction region are accelerated to an energy of some $\mathrm{keV}$ by the potentials applied to two grids. They are detected by a channelplate detector after a drift length of typically $2 \mathrm{~m}$.

With this set-up an overall efficiency of the instrument of $2 \times 10^{-5}$ was found for plutonium corresponding to a detection limit of $4 \times 10^{6}$ per isotope. This is an improvement by two orders of magnitude as compared to the $\alpha$-spectroscopic detection limit $\left(4 \times 10^{8}\right.$ atoms at a measuring time of 16 hours $)$. In addition to be fast, the isotopic composition can be determined with an uncertainty of some percent.

In order to find efficient resonance ionization schemes, detailed spectroscopy (transition probabilities, lifetimes, hyperfine structures, isotope shilts) has to be performed prior to the choice of the optimum resonant ionization path. Furthermore, the ionization potentials have to be determined for the heaviest isotopes. So far, no experimental data were known for the actinides heavier than neptunium [10]. The determination of the ionization potential is possible by use of resonance ionization spectroscopy in an electric field. Figure 7 shows the thresholds where ionization set in for thorium and americium as a function of the squarc-root 


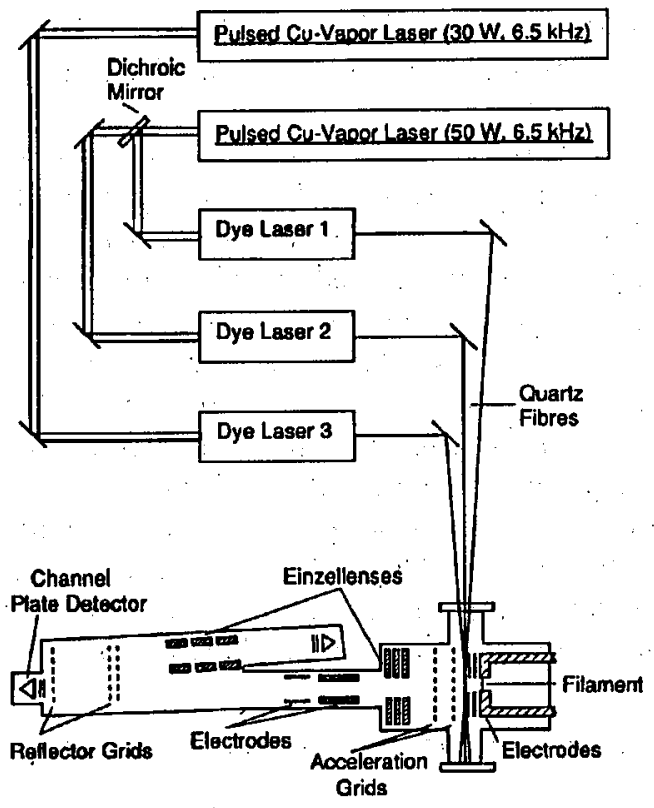

Fig. 6. Set-up for trace analysis of plutonium and other actinides evaporated from a filament. The atoms are resonantly stepwise excited and ionized by the light of three dye lasers pumped by two copper vapour lasers. The ions are mass selectively detected by means of a reflectron type time-of-flight mass spectrometer.

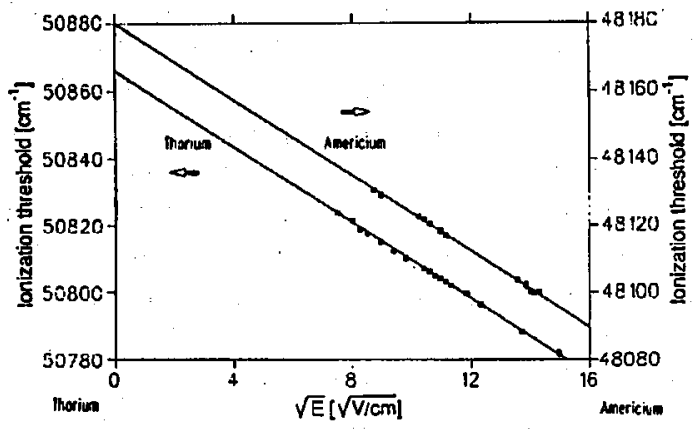

Fig. 7. The ionization threshold of thorium (left scale) and americium (right scale) versus the square root of the electric field $E$ in the interaction region. The data points are fitted by a straight line. Linear extrapolation to $E=0$ yields for thorium an ionization potential (IP) of $6.3067(2) \mathrm{eV}$ and for americium a value of $5.9736(3) \mathrm{eV}$.

of the electric field in the interaction region. As expected from the saddle point model, the data should follow a straight line. Extrapolation to zero electric field yields the ionization potential. In this way the knowledge of the ionization potential has been improved for thorium and neptunium and for the first time determined 
for americium [11]. Due to the high sensitivity of RIMS a sample containing less than $10^{12}$ atoms is sufficient for a determination of the ionization potential.

The total efficiency of RIMS with a copper vapour laser pumped dye laser is of the order of $10^{-5}$. The main losses are due to the temporal overlap $\left(4 \times 10^{-2}\right.$ at $6.5 \mathrm{kHz}$ repetition rate) and the spatial overlap between continuous atomic and pulsed laser beam $\left(6 \times 10^{-2}\right)$. These losses can drastically be reduced by the concept of confinement in a hot cell, i.e. of the laser ion source (see below).

\subsection{Laser ion source}

As shown above, the detection efficiency for atoms in a thermal atomic beam is limited by the poor temporal and spatial overlap of the atomic beam and the laser beams, even at the high repetition rate of copper vapour lasers. These limitations can be significantly reduced by placing the sample into a hot cavity or tube which is provided with a small hole where the laser beams are injected and the photo ions are extracted by an electric field. In this case the atoms will pass the interaction region with the laser light several times before they can escape from the cavity. Hence, an increase in ionization efficiency is expected [12].

At on-line isotope separators, one big problem is to obtain isobarically pure radioactive ions beams. At present, the ion sources most widely used for this purpose are based on surface ionization or ionization in a gas discharge plasma. However, as a result of the thermal character of these processes, the ionization

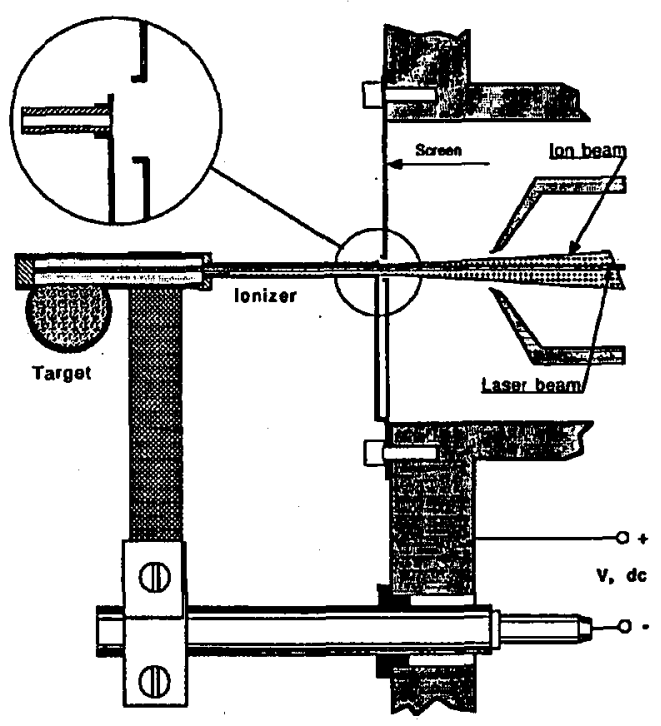

Fig. 8. Scheme of the target, laser ion source and mass separator as used at the on-line isotope separator ISOLDE. The target is bombarded by protons $(2 \mu \mathrm{A}, 600 \mathrm{MeV})$. The reaction products diffuse into the ionizer tube where they are stepwise resonantly photo ionized by the light of three dye lasers pumped by copper vapour lasers. 


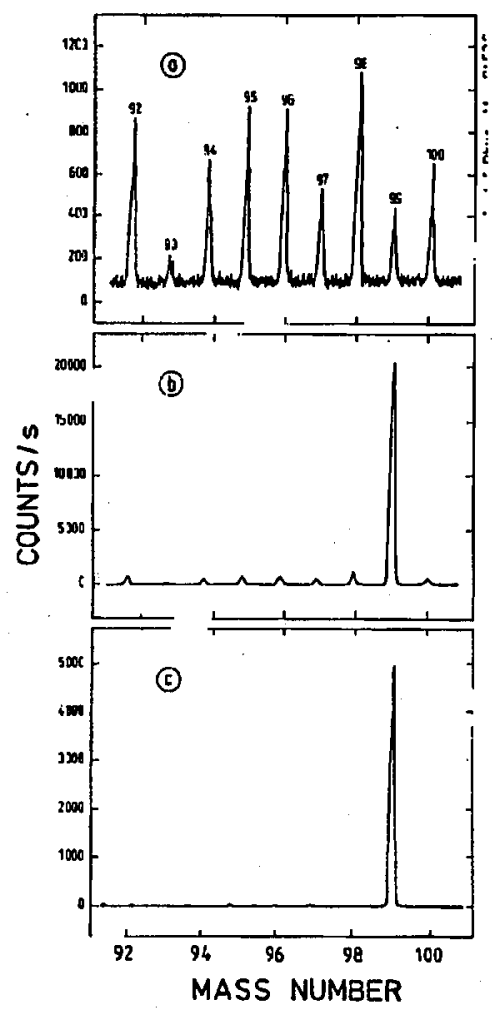

Fig. 9. Mass spectrum of ${ }^{99} \mathrm{Tc}$ and the stable molybdenum isotopes in the range of $92 \leq A \leq 100$. Top: With laser shut off. Middle: With lasers on and in resonance for photo ionization of ${ }^{99} \mathrm{Tc}$. Bottom: The same as before but with gated detection (gate length: $1.5 \mu \mathrm{s}$ ).

is in many cases non-selective with respect to the different elements. Hence, the separated ion beams are contaminated by isobars. This problem can be solved or drastically be reduced by resonance ionization [13].

Figure 8 shows the set-up as used at the on-line isotope separator ISOLDE/CERN [13]. The efficiency of such a device is determined by the rate of photo ionization and the rate of effusion as neutral atom out of the hole of the hot cavity. Typically one calculates the theoretical efficiency of such a laser ion source to be about $20 \%$ when pulsed lasers with a repetition rate of $10 \mathrm{kIIz}$ are used. Experimentally determined efficiencies range up to $15 \%$.

The application of a laser ion source has been proposed also for the determination of technetium in molybdenum ore. Since technetium isotopes are produced by a solar neutrino reaction from molybdenum ore, a determination of the technetium content in this ore would allow for a integral measurement of the solar neutrino flux over the past several million years. Figure 9 shows a preliminary result for ${ }^{99} \mathrm{Tc}$ in a tungsten cavity. The molybdenum isotopes are present in the source as impurity of tungsten, the construction material of the chamber. With 
lasers off (top) only thermal ionization occurs. With lasers on and in resonance for RIS of ${ }^{99} \mathrm{Tc}$ (middle) the molybdenum isotopes are strongly suppressed. With gated detection in addition (bottom) the ratio of ${ }^{99} \mathrm{Tc}$ to ${ }^{98} \mathrm{Mo}$ is improved by a factor of 2400 as compared to thermal ionization.

\section{Laser-induced recombination}

The inverse of resonance ionization is laser-induced recombination (LIREC). Under normal conditions such a process is not observable since there are no free electrons available for recombination. In strong electron plasmas the situation is different: Ilere, spontaneous, single-step radiative electron capture (REC) is observed which leads to a population of states with predominantly low principle quantum number $n$ and subsequently cascades to the ground state. The population can be enforced by laser-stimulated transitions mainly to states of high $n$ since the laser induced electron capture rate incrcases proportional to the third power of the transition wavelength.

The experimental conditions are cspecially favourable when the electron plasma as well as the ion have a small energy spread. This is just the case in the electron cooler of a storage ring: IIere, cold electrons (in the longitudinal direction, "cooled" by the same trick as in collinear spectroscopy) are overlapped with the ion beam and co-propagating together at the same velocity. By Coulomb collisions, the ions circulating in the storage ring with a revolution frequency of about 1 MIIz dissipate their heat into the reservoir of cold, continuously replaced electrons. Cooling times of $\tau \approx 1 \mathrm{~s}$ have been achieved for protons. For highly charged ions, the Coulomb forces become stronger and therefore the cooling time shorter. In this case, $\tau$ is as short as milliseconds.

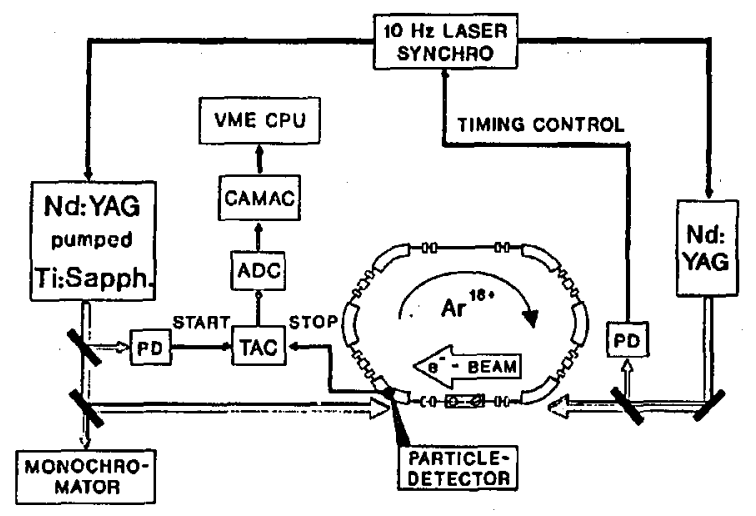

Fig. 10. Two-step resonant laser induced recombination of $\mathrm{Ar}^{18+}$-ions at the storage ring ESR at GSI.

Figure 10 shows the storage ring ESR at GSI/Darmstadt. Ions like bare uranium with energies up to $550 \mathrm{MeV} / \mathrm{u}$ can be injected, cooled by the electron cooler and kept in the ring for hours. The dominant loss process is radiative electron 
capture in the electron cooler. Recently, Beyer et al. [14] took advantage of this effect by measuring the X-rays of $\mathrm{Au}^{79+}$, down-charged to hydrogen-like $\mathrm{Au}^{78+}$. In this way they determined the $1 s$-Lambshift in $\mathrm{Au}^{78+}$ with the until now highest accuracy for heavy ions $(E=212(15 \mathrm{eV}))$. The experimental value is in excellent agreement with theory.

After the first successful demonstration of single-step, laser-induced recombination at the TSR storage ring at Heidelberg for protons and $C^{6+}[15]$ and for protons also in a single-path experiment [16], Borneis et al. observed for the first time the real RIS analogue, the laser-stimulated two-step recombination at the ESR [17]. Figure 10 shows the arrangement. Around $10^{9}$ bare argon ions were injected into the ring and stored. Two pulsed lasers were used: A Nd:YAG laser beam $(\lambda=1.064 \mu \mathrm{m})$ was sent collinearly with the ion beam into the electron cooler. Due to the huge velocity $(\beta=v / c=0.5)$ this laser light was considerably red-shifted in the rest frame of the ion and induced the transition $n=\infty \rightarrow n=81$. Another laser beam (pulsed Ti:Sapphire laser, $760 \mathrm{~nm} \leq \lambda \leq 860 \mathrm{~nm}$ ) was sent anti-collinearly with the ion beam in order to induce transitions from $n=81$ to $n=36$ and 37 (in the ion rest frame around $\lambda=460 \mathrm{~nm}$ ). The LIREC resonance was observed by detecting the down-charged ion $\left(\mathrm{Ar}^{17+}\right)$ in a detector downstream the cooler in the first bending magnet in a time window with an appropriate delay in respect to the laser pulse.

Such investigations will not only enable one to study relativistic and QED effects in the strong fields of highly charged ions but also serve as a diagnostic tool for the beam quality in storage rings and the very peculiar situation of a highly charged ions in a cloud of cold electrons travelling at the same speed as the ion.

\section{Conclusion}

Resonance ionization mass spectroscopy has been proved to be a powerful tool in atomic spectroscopy, tracc analysis, and the investigation of radioactive isotopes. Detailed atomic spectroscopy can be performed on samples containing less than $10^{12}$ atoms. This is especially important for the investigation of atomic properties of transuranium elements. The extremely low detection limit gives access even to samples in the sub-femtogram regime. High elemental and isotopic selectivity can be obtained. The laser ion source can be used in order to obtain isobarically pure ion beams. When inexpensive, powerful and easy to handle laser will become available, the combination of mass spectrometry and resonance ionization spectroscopy will become one of the most powerful techniques in trace analysis.

\section{Acknowledgment}

I would like to thank the many colleagues and co-workers whose material I have used in this publication. The work on short-lived gold and platinum isotopes has been supported by the Bundesminister für Forschung und Technologie. The experiments for fast detection of ${ }^{90,89} \mathrm{Sr}$ and the trace analysis of actinides were funded by the Deutsches Bundesministerium für Umwelt, Naturschutz und Reaktorsicherheit. The laser ion source was developed by a grant of the Deutsche 
Forschungsgemeinschaft. I thank R. Neumann, T. Kühl and co-workers for allowing me to use their material prior to publication. I especially would like to acknowledge the contributions of G. Bollen, G. Passler, and K. Wendt to the work presented in this publication.

\section{References}

[1] V.S. Letokhov, Laser-Photoionization Spectroscopy, Academic Press, London 1987.

[2] G.S. Hurst, M.G. Payne, Principles and Applications of Resonance Ionisation Spectroscopy, Adam Hilger, Bristol, Philadelphia 1988.

[3] Proc. Internat. Symposium on Resonance Ionization, Spectroscopy and its Applications, Institute of Physics Conference Series, Institute of Physics, Bristol, UK.

[4] J. Bonn, G. Huber, II.-J. Kluge, L. Kugler, E.W. Otten, Phys. Lett. B 38, 308 (1972).

[5] K. Wallmeroth, G. Bollen, A. Dohn, P. Egelhof, U. Krönert, M.J.G. Borge, J. Campos, A. Rodriguez-Yunta, K. Heyde, C. De Coster, J.L. Wood, H.-J. Kluge, the ISOLDE Collaboration, Nucl. Phys. A 493, 224 (1989).

[6] Th. Hilberath, St. Becker, G. Bollen, H.-J. Kluge, U. Krönert, G. Passler, J. Rikovska, R. Wyss, the ISOLDE Collaboration, Z. Phys. A 342, 1 (1992).

[7] U. Krönert, St. Becker, G. Bollen, M. Gerber, Th. Hilberath, H.-J. Kluge, G. Passler, the ISOLDE Collaboration, Nucl. Instrum. Methods Phys. Res. A 300, 522 (1991).

[8] L. Monz, R. Hohmann, H.-J. Kluge, S. Kunze, J. Lantzsch, E.-W. Otten, G. Passler, P. Senne, J. Stenner, K. Stratmann, K. Wendt, K. Zimmer, G. Herrmann, N. Trautmann, K. Walter, Spectrochim. Acta B 48, 1655 (1993).

[9] W. Ruster, F. Ames, H.-J. Kluge, E.W. Otten, D. Rehklau, F. Scheerer, G. Herrmann, C. Mühleck, J. Riegel, II. Rimke, P. Sattelberger, N. Trautmann, Nucl. Instrum. Methods Phys. Res. A 281, 547 (1989).

[10] J. Riegel, R. Deißenberger, G. Herrmann, P. Sattelberger, N. Trautmann, H. Wendeler, F. Ames, H.-J. K'luge, F. Scheerer, F.J. Urban, Appl. Phys. B 56, 273 (1993).

[11] R. Deissenberger, F. Amcs, G. Herrmann, H.-J. Kluge, S. Köhler, G. Passler, J. Riegel, F. Scheerer, N. Trautmann, F.-J. Urban, H. Wendeler, to be published.

[12] F. Scheerer, F. Albus, F. Ames, II.-J. Kluge, N. Trautmann, Spectrochim. Acta B 47, 793 (1992).

[13] V.I. Mishin, V.N. Fedoseyev, II.-J. K'luge, V.S. Letokhov, H.L. Ravn, F. Scheerer, Y. Shirakabe, S. Sundell, O. Tengblad, the ISOLDE Collaboration, Nucl. Instrum. Methods Phys. Res. B 73, 550 (1993).

[14] H.F. Beyer, D. Liesen, F. Bosch, K.D. Finlayson, M. Jung, O. Klepper, R. Moshammer, K. Beckert, H. Eickhoff, B. Franzke, F. Nolden, P. Spädtke, M. Steck, G. Menzel, R.D. Deslattes, Phys. Lett. A 184, 435 (1994).

[15] U. Schramm, J. Berger, M. Grieser, D. Ilabs, E. Jaeschke, G. Kilgus, D. Schwalm, A. Wolf, R. Neumann, R. Schuch, Phys. Rev. Lelt. 67, 22 (1991).

[16] F.B. Yousif, P. Van der Donk, Z. Kucherovski, J. Reis, E. Brannen, J.B.A. Mitchell, T.J. Morgan, Phys. Rev. Lelt. 67, 26 (1991).

[17] S. Borneis, F. Bosch, T. Engel, I. Klaft, O. Klepper, T. Külıl, D. Marx, R. Moshammer, R. Neumann, S. Schröder, P. Seelig, L. Völker, Phys. Rev. Lett. 72, 207 (1994). 